\title{
Method for hybrid recording with single layer CoTbAg medium
}

\author{
C. T. Lie, ${ }^{\text {a) }}$ P. C. Kuo, and C. L. Shen \\ Institute of Materials Science and Engineering, National Taiwan University, Taipei 106, Taiwan
}

(Received 14 February 2003; accepted 7 June 2003)

This work presents a hybrid-recording disk with a single layer CoTbAg recording medium. $\mathrm{Co}_{69.48-x} \mathrm{~Tb}_{30.52} \mathrm{Ag}_{x}$ films with $x=0-25.68$ at. $\%$ are fabricated on glass and naturally oxidized silicon wafer substrate by dc magnetron sputtering. The effects of $\mathrm{Ag}$ content on the magnetic properties and the microstructure of the film are investigated. The transmission electron microscope diffraction pattern indicates that all the films are amorphous. The saturation magnetization of the $\mathrm{Co}_{67.23} \mathrm{~Tb}_{30.52} \mathrm{Ag}_{2.25}$ film is about $310 \mathrm{emu} / \mathrm{cm}^{3}$; perpendicular remanence is about $255 \mathrm{emu} / \mathrm{cm}^{3}$, and the perpendicular coercivity is about 3100 Oe at room temperature. The saturation magnetization and perpendicular coercivity decrease rapidly as temperature increases from room temperature to $200{ }^{\circ} \mathrm{C}$. The compensation temperature $T_{\text {comp }}$ of this film is about $225^{\circ} \mathrm{C}$. This film is a promising candidate for hybrid recording media applications. (c) 2003 American Institute of Physics.

[DOI: $10.1063 / 1.1595706]$

\section{INTRODUCTION}

The hybrid recording method with perpendicular thermo-magnetic writing and magnetic flux reading was recently proposed to increase the recording density of the magnetic disk. ${ }^{1,2}$ This method enables perpendicular magnetic domains to be formed in an amorphous film medium and utilizes a high-sensitivity magnetic flux detector [for example, a giant magnetoresistive (GMR) head or a tunneling magnetoresistive (TMR) head]. The formation of smaller recording domains is the key issue to this method if the recording density wants to increase further.

Using near-field optics, the solid immersion lens (SIL) has been used to shrink the laser spot size to reduce the domain size. ${ }^{3}$ However, such near-field recording requires the distance between the SIL and the surface of the disk to be less than the light wavelength $\lambda$. Contact between the optical lens and the surface of the disk during recording cannot be avoided. The SIL becomes failed as it contacts with the lubricant on the magnetic disk surface.

Guerra et al. proposed a near-field optical data storage disk, the integral near field optics (INFO) disk. ${ }^{4,5}$ The SIL cylinder is made circular on the PC substrate of a digital versatile disk (DVD) as the recording track. The track density of this disk can exceed that of a conventional DVD. ${ }^{4}$ The numerical aperture (NA) depends on the refractive index of the SIL cylinder.

Figure 1 shows the proposed design called the "nearfield hybrid recording without low-flying optical head," which combines the advantages of an INFO disk and hybrid recording: the SIL cylinder is made on the substrate of the recording disk to avoid contact between the optical lens and the magnetic disk surface. And the magnetic recording layer can be closer to the magnetic writer/reader. The SIL cylinder in Fig. 1 is $\mathrm{ZnS}: \mathrm{SiO}_{2}$, its effective NA is about 1.1. The recording medium is single layer CoTbAg film.

a) Author to whom correspondence should be addressed; Electronic mail: f86542011@ntu.edu.tw
This design replaces the currently used doubly layered medium ${ }^{6,7}$ by a single layer recording medium. A single layered recording medium for hybrid recording must satisfy two requirements: it must provide satisfactory magneto-optical (MO) writing performance and large saturation magnetization $M_{s}$ that generates sufficient magnetic flux for GMR or TMR head readout. The $M_{s}$ value of the traditional MO medium is very small near room temperature. ${ }^{8,9}$ In a previous study, we have shown that the perpendicular coercivity of the amorphous $\mathrm{CoTb}$ film is very high at room temperature but its $M_{s}$ value is small. ${ }^{10}$ This study shows that the single layer $\mathrm{Co}_{69.48-x} \mathrm{~Tb}_{30.52} \mathrm{Ag}_{x}$ film with a little $\mathrm{Ag}$ content is suitable for hybrid recording media applications. The effects of $\mathrm{Ag}$ content on the structure and magnetic properties of $\mathrm{Co}_{69.48-x} \mathrm{~Tb}_{30.52} \mathrm{Ag}_{x}$ films are also investigated.

\section{EXPERIMENT}

$\mathrm{Co}_{69.48-x} \mathrm{~Tb}_{30.52} \mathrm{Ag}_{x}$ films (where $x=0-25.68$ at. \%) were deposited on glass and natural-oxidized $\mathrm{Si}(100)$ wafer substrates by dc magnetron sputtering at room temperature.

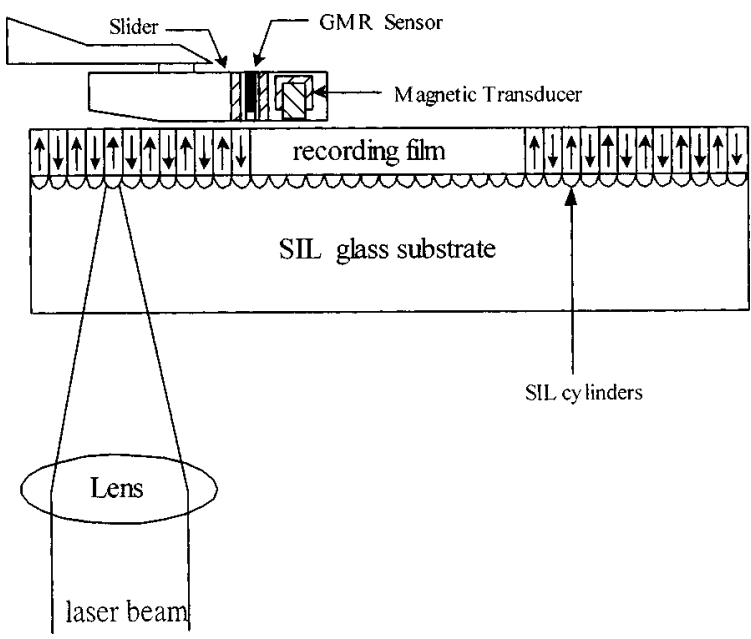

FIG. 1. Configuration of the disk tester. 


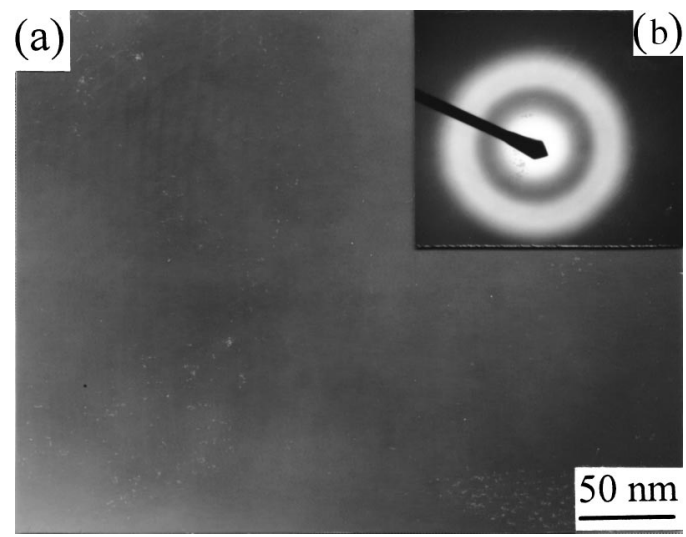

FIG. 2. (a) TEM bright field image and (b) electron diffraction pattern of the $\mathrm{Co}_{43.8} \mathrm{~Tb}_{30.52} \mathrm{Ag}_{25.68}$ film.

The target was a Co disk overlaid with $\mathrm{Tb}$ and $\mathrm{Ag}$ pieces, to yield the desired film composition. The magnetic film was sandwiched between $\mathrm{SiN}_{x}$ protective layers to prevent oxidation. The $\mathrm{SiN}_{x}$ protective layer was prepared by rf magnetron sputtering of the $\mathrm{Si}_{3} \mathrm{~N}_{4}$ target. The thickness of the magnetic layer and the protective layer was 75 and $30 \mathrm{~nm}$, respectively.

The structure of the film was examined by transmission electron microscope (TEM). The composition of the film was determined by energy disperse spectroscopy. The depth profiles of the elements in the film were analyzed by Auger electron spectroscopy (AES). The film thickness was measured by atomic force microscopy and $\alpha$-step. The magnetic properties of the films were measured by a vibrating sample magnetometer with a maximum applied field of $13 \mathrm{kOe}$.

\section{RESULTS AND DISCUSSION}

The CoTbAg films were examined by TEM to understand the effect of $\mathrm{Ag}$ content on the structure of the amor-

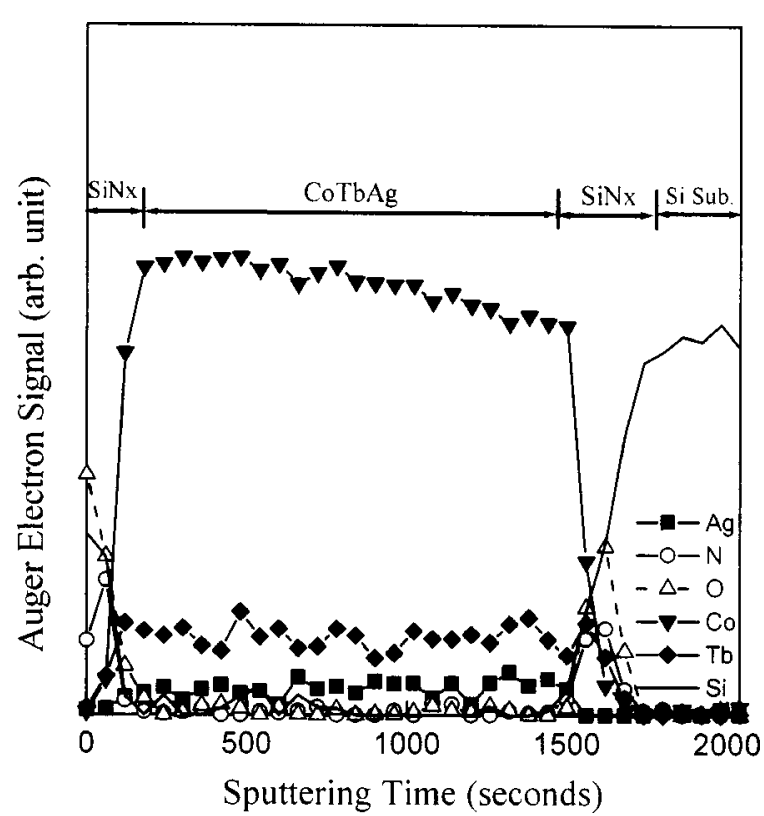

FIG. 3. AES depth profiles of the elements in the $\mathrm{Co}_{67.23} \mathrm{~Tb}_{30.52} \mathrm{Ag}_{2.25}$ film.
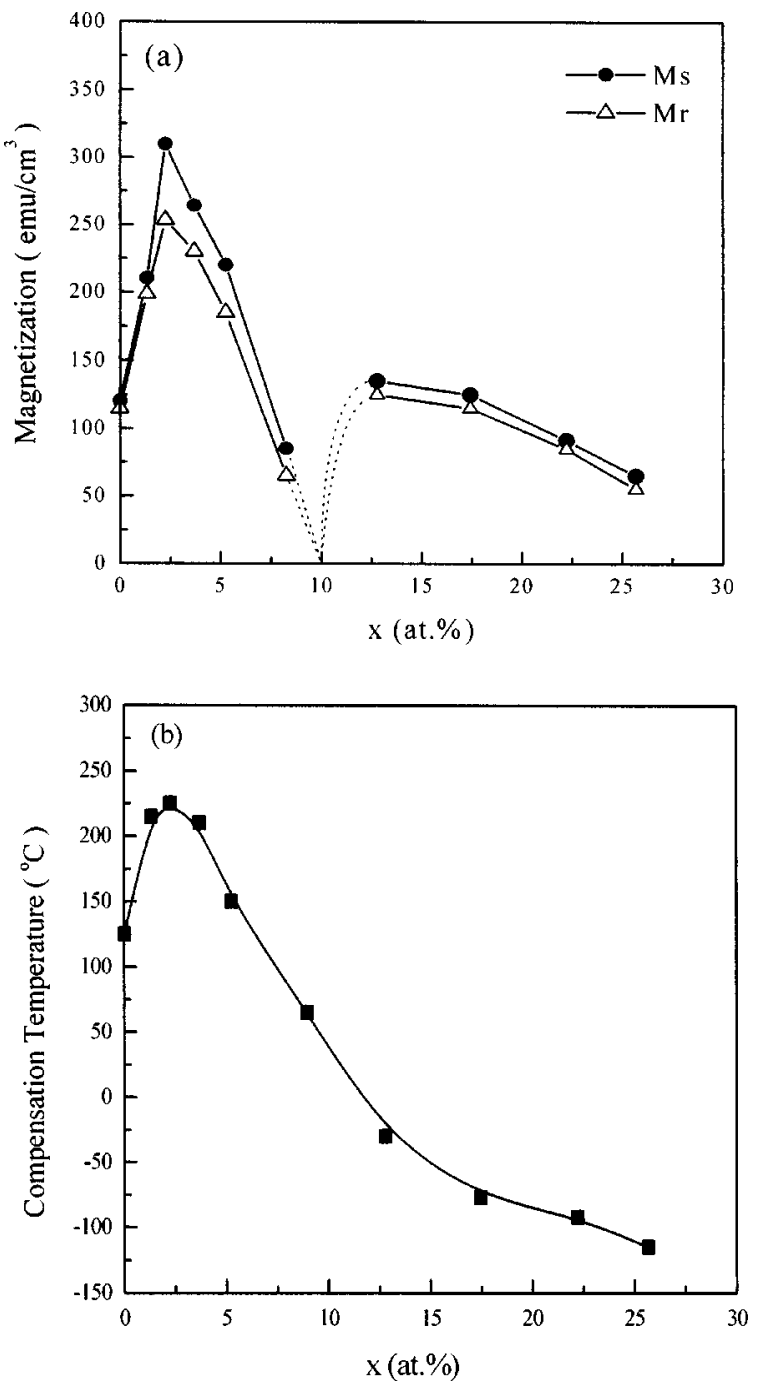

FIG. 4. (a) Variations of $M_{s}$ and $M_{r}$ with $\mathrm{Ag}$ content of the $\mathrm{Co}_{69.48-x} \mathrm{~Tb}_{30.52} \mathrm{Ag}_{x}$ film and (b) relationship between $T_{\text {comp }}$ and $\mathrm{Ag}$ content of the $\mathrm{Co}_{69.48-x} \mathrm{~Tb}_{30.52} \mathrm{Ag}_{x}$ film.

phous $\mathrm{CoTb}$ film. It was found that all the $\mathrm{Co}_{69.48-x} \mathrm{~Tb}_{30.52} \mathrm{Ag}_{x}$ films $(x=0-25.68$ at. \%) were amorphous. Figure 2(a) shows a TEM bright field image of the $\mathrm{Co}_{43.8} \mathrm{~Tb}_{30.52} \mathrm{Ag}_{25.68}$ film and Fig. 2(b) is the electron selected area diffraction (SAD) pattern of Fig. 2(a). Figure 2(a) shows no crystal grains and the shape of the SAD pattern is a broad halo, implying that the film is amorphous.

Figure 3 shows the depth profiles of elements in the $\mathrm{Co}_{67.23} \mathrm{~Tb}_{30.52} \mathrm{Ag}_{2.25}$ film. It can be seen that a $30 \mathrm{~nm}$ thick SiNx protective layer effectively prevents the oxidization of the magnetic film. Some oxygen is present in the SiNx protective layers and in the surface of the naturally oxidized $\mathrm{Si}$ substrate but not in the magnetic layer.

Figure 4(a) shows the variations of saturation magnetization $\left(M_{s}\right)$ and perpendicular remanence $\left(M_{r}\right)$ with $\mathrm{Ag}$ content of the $\mathrm{Co}_{69.48-x} \mathrm{~Tb}_{30.52} \mathrm{Ag}_{x}$ film at room temperature. It reveals that the $M_{s}$ and $M_{r}$ values of the $\mathrm{Co}_{69.48} \mathrm{~Tb}_{30.52}$ film $(\mathrm{x}=0$ at. $\%)$ are about 125 and $115 \mathrm{emu} / \mathrm{cm}^{3}$, respectively. The variation of the squareness $\left(M_{r} / M_{s}\right)$ with $\mathrm{Ag}$ content is very small and the squareness is higher than 0.8 for all the films. $M_{s}$ and $M_{r}$ increase rapidly with Ag content as $x$ 


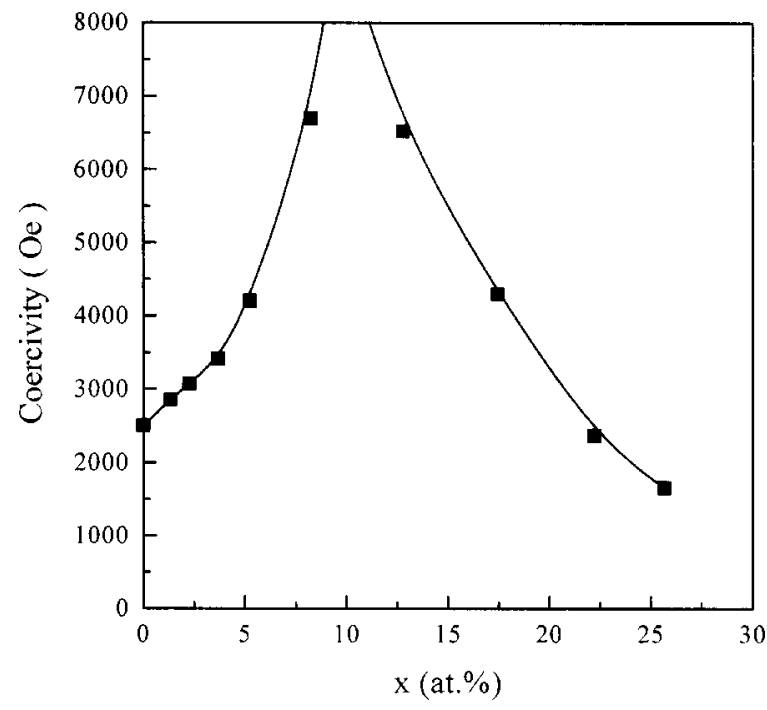

FIG. 5. Variation of $H_{c}$ with $\mathrm{Ag}$ content of the $\mathrm{Co}_{69.48-x} \mathrm{~Tb}_{30.52} \mathrm{Ag}_{x}$ film.

$<2.25$ at. $\%$ and then decrease rapidly with increasing $\mathrm{Ag}$ content as $x>2.25$ at. \%. The maximum $M_{s}$ and $M_{r}$ values occur at $x \sim 2.25$ at. \%: they are about 310 and $255 \mathrm{emu} / \mathrm{cm}^{3}$, respectively. In the $\mathrm{Co}_{69.48-x} \mathrm{~Tb}_{30.52} \mathrm{Ag}_{x}$ alloy system, $\mathrm{Ag}$ is a nonmagnetic element. The saturation magnetizations of $\mathrm{Co} / \mathrm{Pd}^{11,12}$ and $\mathrm{Fe} / \mathrm{Ag}^{13}$ multilayers have been shown to exceed those of pure Co and Fe films, respectively, because of the spin polarization of nonmagnetic layers. However, the spin polarization of the $\mathrm{Ag}$ element is not enough to enhance the $M_{s}$ value from 125 to $310 \mathrm{emu} / \mathrm{cm}^{3}$ after addition of 2.25 at. $\% \mathrm{Ag}$ to $\mathrm{CoTb}$. The variation of $M_{s}$ value with $\mathrm{Ag}$ content follows not only from the spin polarization of $\mathrm{Ag}$ atoms but also from the shift of compensation temperature $T_{\text {comp }}$. $T_{\text {comp }}$ of the rare earth-transition metal (RE-TM) alloy is very sensitive to the composition of the alloy. ${ }^{14}$ Figure 4(b) shows the relationship between $T_{\text {comp }}$ and $\mathrm{Ag}$ content of the $\mathrm{Co}_{69.48-x} \mathrm{~Tb}_{30.52} \mathrm{Ag}_{x}$ film. The relationship between $T_{\text {comp }}$ and $\mathrm{Ag}$ content is not linear. $T_{\text {comp }}$ increases with $\mathrm{Ag}$ content as $x$ increases to 2.25 at. \% but decreases as $\mathrm{Ag}$ content is increased further. $T_{\text {comp }}$ of the $\mathrm{Co}_{69.48} \mathrm{~Tb}_{30.52}$ film ( $x$ $=0$ at. \%) is about $125^{\circ} \mathrm{C}$, and increases to $225^{\circ} \mathrm{C}$ as $\mathrm{Ag}$ content is increased to 2.25 at. \%. $T_{\text {comp }}$ then decreases as $\mathrm{Ag}$ content increases, as shown in Fig. 4(b).

Figure 5 shows the variation of perpendicular coercivity Hc with $\mathrm{Ag}$ content of the $\mathrm{Co}_{69.48-x} \mathrm{~Tb}_{30.52} \mathrm{Ag}_{x}$ film at room temperature. The $H_{c}$ value increases from about 2500 to $7200 \mathrm{Oe}$ as $\mathrm{Ag}$ content increases from 0 to 8.26 at. \%, and $H_{c}$ decreases rapidly with increasing $\mathrm{Ag}$ content when $x$ $>10$ at. \%. At room temperature, $M_{s}$ is zero and $H_{c}$ is infinite at the compensation composition. Figure 4(b) shows that the compensation composition of the $\mathrm{Co}_{69.48-x} \mathrm{~Tb}_{30.52} \mathrm{Ag}_{x}$ film is $x \sim 10$ at. \% . Consequently, the $\mathrm{Co}_{69.48-x} \mathrm{~Tb}_{30.52} \mathrm{Ag}_{x}$ alloy is transformed from $\mathrm{RE}$ rich to $\mathrm{TM}$ rich at $x$ $\sim 10$ at. $\%$ at room temperature. In the TM-rich region $(x$ $>10$ at. $\%), H_{c}$ decreases as $T_{\text {comp }}$ is decreased. In the RErich region $\left(x<10\right.$ at. \%), $M_{s}$ increases, but $H_{c}$ decreases as $T_{\text {comp }}$ is increased. However, in Figs. 4(a) and 5, both $M_{s}$ and $H_{c}$ increase with $x$ when $\mathrm{Ag}$ content is less than 2.25 at. \%. We conjecture the discrepancy between $H_{c}$ and $T_{\text {comp }}$ when
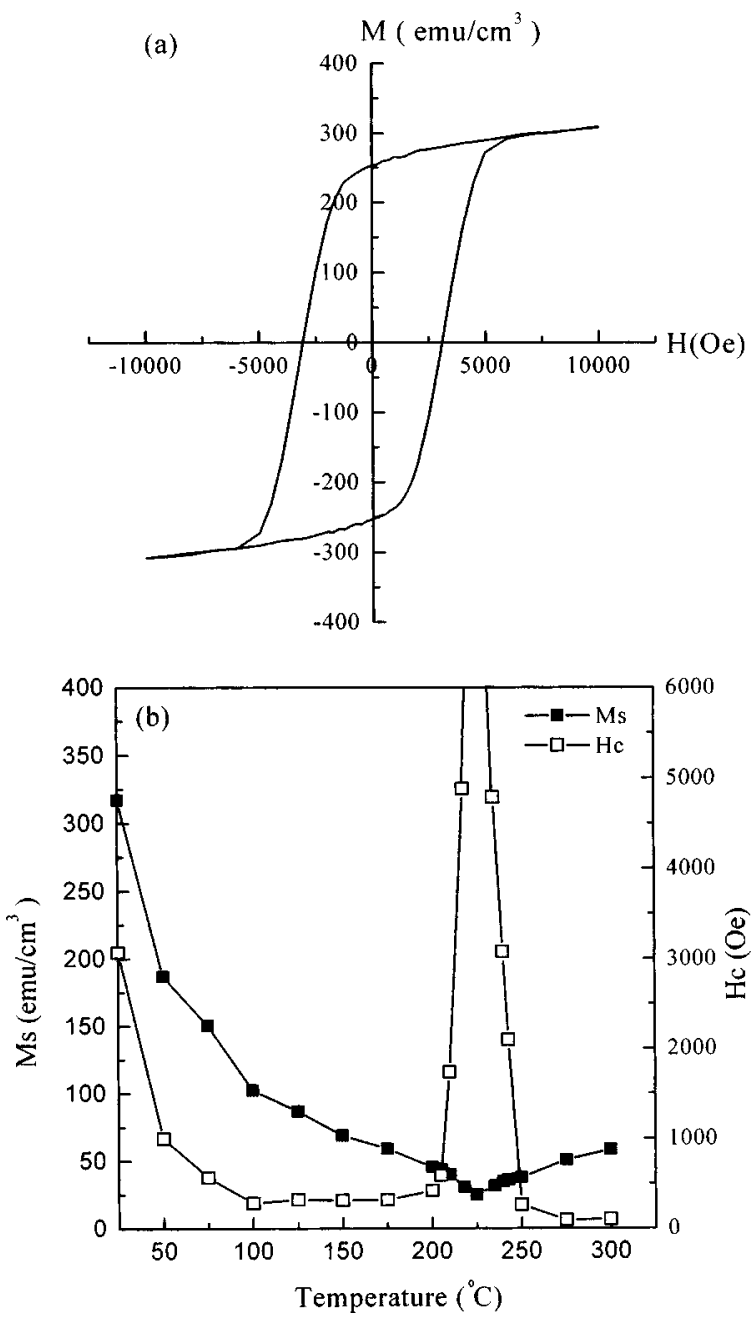

FIG. 6. (a) $M-H$ loop of the $\mathrm{Co}_{67.23} \mathrm{~Tb}_{30.52} \mathrm{Ag}_{2.25}$ film and (b) variations of $M_{s}$ and $H_{c}$ with temperature of the $\mathrm{Co}_{67.23} \mathrm{~Tb}_{30.52} \mathrm{Ag}_{2.25}$ film.

$x<2.25$ at. \% is related to some $\mathrm{Ag}$ atoms are close forming clusters in the CoTbAg film. (From Fig. 3, it can be seen that the distribution of $\mathrm{Ag}$ in the CoTbAg film is not uniform.) These clusters are like defects that impede domain wall motion to reduce the domain wall energy, pinning the domain wall, and increasing the coercivity of the film. ${ }^{15}$

Figure 6(a) is the $M-H$ loop of the $\mathrm{Co}_{67.23} \mathrm{~Tb}_{30.52} \mathrm{Ag}_{2.25}$ film at room temperature, where the applied filed is perpendicular to the film plane. The saturation magnetization of this film is about $310 \mathrm{emu} / \mathrm{cm}^{3} ; M_{r}$ is about $255 \mathrm{emu} / \mathrm{cm}^{3}$, and the perpendicular coercivity is about 3100 Oe. Figure 6(b) shows the variations of $M_{s}$ and $H_{c}$ with temperature of this film, with a silicon substrate. $M_{s}$ of the film decreases from 310 to about $25 \mathrm{emu} / \mathrm{cm}^{3}$ as the temperature increases from 25 to $225^{\circ} \mathrm{C}$ and $M_{s}$ increases with temperature above $225^{\circ} \mathrm{C}$, indicating that the $T_{\text {comp }}$ of this film is about $225^{\circ} \mathrm{C}$. Its $H_{c}$ decreases from 3100 to about $300 \mathrm{Oe}$ as the temperature increases from 25 to $100^{\circ} \mathrm{C}$, then remains at this low $H_{c}$ as temperature is further increased to $200^{\circ} \mathrm{C}$. $H_{c}$ increases rapidly with temperature from 200 to $225^{\circ} \mathrm{C}$ and $H_{c}$ decreases rapidly to about $250 \mathrm{Oe}$ as temperature increases from 225 to $250{ }^{\circ} \mathrm{C}$. This film can be a promising candidate for hybrid recording media. 


\section{ACKNOWLEDGMENTS}

This work was supported by the National Science Council and Ministry of Economic Affairs of Taiwan, R.O.C., through Grant Nos. NSC 90-2216-E 002-036 and MOEA 91-EC-17-A-08-S1-0006, respectively.

${ }^{1}$ J. J. M. Ruigrok, R. Coehoorn, S. R. Cumpson, and H. W. Kesteren, J. Appl. Phys. 87, 5398 (2000).

${ }^{2}$ H. Saga, H. Nemoto, H. Sukeda, and M. Takahashi, Jpn. J. Appl. Phys., Part 1 38, 1839 (1999).

${ }^{3}$ A. Chekanov, M. Birukawa, Y. Itoh, and T. Suzuki, IEEE Trans. Magn. 35, 3100 (1999).

${ }^{4}$ J. Guerra, D. Vezenov, P. Sullivan, W. Haimberger, and L. Thulin, Jpn. J. Appl. Phys., Part 1 41, 1866 (2002).

${ }^{5}$ J. M. Guerra, U.S. Patent No. 5,910,940 (8 June, 1999).
${ }^{6}$ H. Nemoto, H. Saga, H. Sukeda, and M. Takahashi, Jpn. J. Appl. Phys., Part 1 38, 1841 (1999).

${ }^{7}$ C.-C. Lin, C.-H. Lai, B. M. Chen, and H.-P. D. Shieh, IEEE Trans. Magn. 37, 1339 (2001)

${ }^{8}$ S. Takayama, T. Niihara, K. Kaneko, Y. Sugita, and M. Ojima, J. Appl. Phys. 61, 2610 (1987)

${ }^{9}$ M. Takahashi, T. Niihara, and N. Ohta, J. Appl. Phys. 64, 262 (1988).

${ }^{10}$ P. C. Kuo and C. M. Kuo, J. Appl. Phys. 84, 3317 (1998).

${ }^{11}$ F. J. A. den Broeder, H. C. Donkersloot, H. J. G. Draaisma, and W. J. M. de Jonge, J. Appl. Phys. 61, 4317 (1987).

${ }^{12}$ K. Miura, H. Kimura, S. Imanaga, and Y. Hayafuji, J. Appl. Phys. 72, 4826 (1992)

${ }^{13}$ Y. B. Xu et al., J. Appl. Phys. 75, 6190 (1994).

${ }^{14}$ P. Hansen, S. Klahn, C. Clausen, G. Much, and K. Witter, J. Appl. Phys. 69, 3194 (1991).

${ }^{15}$ The Physical Principles of Magneto-optical Recording, edited by M. Mansuripur (Cambridge University Press, Cambridge, 1995), p. 46. 